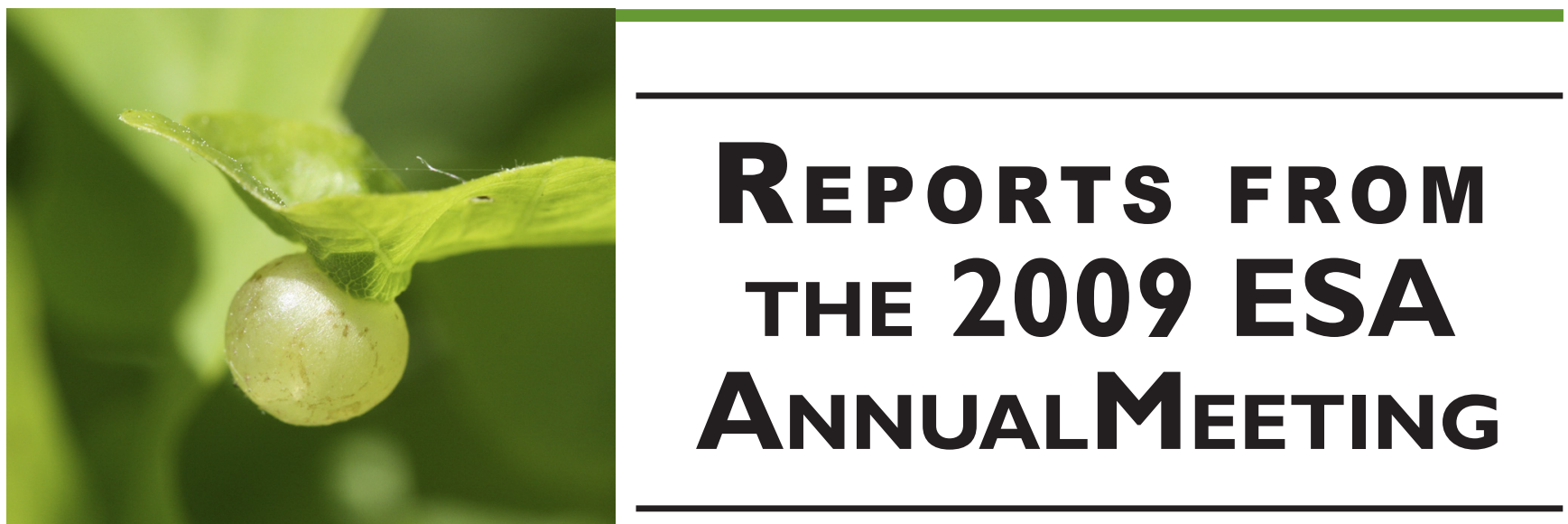

\title{
Symposium 6: Mutualistic Networks
}

Symposium 6 was organized by J. A. Bascompte, moderated by M. A. Fortuna, and held during the ESA Annual Meeting in Albuquerque, New Mexico, on 4 August 2009. The participants are listed at the end of this report.

In recent years the theory of complex networks has allowed us to systematically address the complexities of entire mutualistic networks. This is one of the most fascinating topics in community ecology and coevolution, as the architecture of the mutualistic interactions between plants and animals has considerable implications for community robustness, conservation biology, and the coevolutionary process.

The first stage of research in mutualistic networks focused on describing their structure. These networks have been found to be very heterogeneous (while the bulk of species have a few interactions, a few species are much more connected than expected by chance); nested (specialists interact with species that form well-defined subsets of the species that generalists interact with); and built upon weak and asymmetric links among species. Thus, mutualisms are not randomly organized within species-rich communities.

Currently, ecologists and evolutionary biologists are moving beyond this descriptive first stage to start unraveling the dynamic mechanisms and consequences of network structure. This recent work is reviewed in this symposium, with the ultimate goal of increasing information flow and stimulating discussion. To meet this aim we invited a sample of the most active ecologists in this area. These ecologists were from eight countries, worked on different types of organisms, and used different approaches. Specific questions included comparisons across different interaction types (mutalistic vs. antagonistic), the magnitude of phylogenetic signal in network patterns, the dynamics of networks, and implications for community robustness and conservation.

Similarities and differences across interaction types

Colin Fontaine compared the structure of mutualistic and antagonistic networks. His talk was 
motivated by recent studies highlighting structural differences between networks depending on the type of ecological interaction involved, i.e., mutualistic or antagonistic. He addressed these questions using a comparative network approach between plant-pollinator and plant-herbivore communities. As he showed, trophic and mutualistic networks actually differ in their structure. Pollination networks appear more connected, more nested, and less modular than herbivory networks. These differences were explained in the light of the dynamic analysis of models of mutualistic vs. models of antagonistic interactions (see General discussion).

\section{Process-generating network patterns}

Pedro Jordano showed that the overall interaction pattern in two highly sampled frugivorous networks in Southern Spain is influenced by the plant phylogeny, suggesting that the plant assemblage drives the interaction. Plant-frugivore coevolution is suggested to be a process of resource tracking by frugivores, so that each plant species "filters out" subsets of frugivore species given species-specific traits that constrain their interaction.

Victor Rico-Gray, in turn, reviewed work analyzing the structure of a multispecific network of interacting ants and extrafloral-nectary-bearing plants in La Mancha, Veracruz, Mexico. He found that interaction intimacy (i.e., the degree of biological association among partners: symbiotic vs. nonsymbiotic) markedly affects the structure of these networks. When interaction intimacy is high, the networks are compartmentalized, while they are nested when interaction intimacy is low. Victor emphasized that these results support a promising approach for the development of multispecies coevolutionary theory, leading to the idea that specialization may coevolve in different but simple ways in antagonistic and mutualistic assemblages, with important consequences for the organization of biodiversity.

\section{Temporal dynamics}

Most mutualistic networks are static and based on data aggregation through time and space. However, a few recent studies describe the change in structural properties during a single season or a few years. Both Jens Olesen and Theodora Petanidou reviewed their research on network dynamics. They both showed a high degree of temporal variability in species composition and interactions, and yet a constant network structure. Theodora showed that floral prototypes preferred by flower-visiting groups (i.e., the so called "floral syndromes") vary from year to year. Jens showed that species going extinct or invading the habitats follow specific topological trajectories. These temporal studies offer a unique opportunity to track how external factors influence an ecological network. A similar conclusion was also reported by Victor Rico-Gray, who found that the nested structure of ant-plant networks remained constant in time despite increases in the presence of invasive or ruderal species.

Implications for network robustness and size

Jordi Bascompte reviewed some theoretical work exploring the dynamic implications of network structure. Several studies conclude that the structure of mutualistic networks makes them more robust to the random extinction of species, but makes them very fragile to the extinction of the most generalist species forming the core of the nested network. When phylogenetic information is 
added, the rate of evolutionary history lost is higher than expected due to the phylogenetic signal on network patterns. Second, analytic models of community dynamics have found that nested networks reduce the competitive load and increase the number of coexisting species. Similarly, Colin Fontaine explored the degree to which the type of ecological interaction (mutualistic vs. antagonistic) affects the relationship between network structure and its persistence and stability. Thus, in mutualistic networks, both low modularity and high connectance promote stability. The complete opposite pattern was observed in trophic networks, where low nestedness and low connectance enhance stability. Strikingly, network characteristics that appear to promote stability in each type of interaction model are also the ones that are mostly observed in each type of empirical interaction network. This congruence between theoretical predictions and empirical data suggests that stability and coexistence constraints might play an important role in shaping the singular architecture of mutualistic networks.

\section{Consequences for global change}

Global change has been shown to have pervasive effects on the disruption of mutualisms. What are the practical implications of network structure for conservation? Jane Memmott advocated that the interactions between species can provide a valuable metric when considering the efficacy of ecological restoration programs. She illustrated this point with three successful restoration programs. Among her results, it was shown that the removal of alien plants could lead to the loss of an important food supply of the native pollinators, with potentially detrimental effects on one of the focal native plants, at least in the short term. Also, parasites can be excellent markers of restoration success, as they are some of the last species to return to the community. These examples put network theory to the test in the real world.

\section{General discussion: the way forward}

As subjects for the future, there is almost no work addressing the spatial dimension of entire networks. That is, how are networks organized through space? In short, this calls for an extension of island biogeography, addressing interactions beyond the number of species. An integrated framework provided by (1) metacommunity theory, defined as a set of spatially coupled local communities, and (2) the geographic mosaic theory, dealing with the structure of (reciprocal) selection across species, can successfully address this question. An island biogeographic approach could also provide insight into the rate and shape of network collapse as a consequence of habitat fragmentation.

A second gap in network studies is provided by the fact that almost all studies consider only one interaction type (antagonistic or mutualistic), and therefore we know almost nothing about how different interaction types are combined within an ecological community. Work in this area may provide a better assessment on how the stability of communities depends on the way several interaction types are combined to form a single ecological network. A better understanding of the coevolutionary meaning of an interaction is also necessary.

Finally, we asked ourselves what the most relevant contribution of applying network theory to studies of coevolution may be. As with any other theoretical framework, there may be a component of fad in studying networks, and therefore it is important to think critically about what we have gained from a network approach that has significantly advanced our ecological knowledge about mutualisms, 
besides rephrasing old facts with a new jargon. Arguably, the network approach has put the emphasis on the community context of mutualism, and provides a means of tackling complexity in a community context. Several approaches to studying coevolution within networks are beginning to develop. These include analyses of how smaller multispecific modules form and coevolve as geographic mosaics across space and time within larger networks, and how coevolution may indirectly, as well as directly, shape the topology of networks. Useful methodological approaches to fulfill these analyses are phylogenetic statistical tools and adaptive dynamic approaches.

Jordi Bascompte (Estación Biológica de Doñana, CSIC, Sevilla, Spain. Corresponding author. E-mail: bascompte@ebd.csic.es)

Marcelo Aizen (Universidad Nacional del Comahue, Bariloche, Argentina)

Colin Fontaine (Imperial College, Silwood Park, Ascot, UK)

Miguel A. Fortuna (Princeton University, Princeton, New Jersey, USA)

Pedro Jordano (Estación Biológica de Doñana, CSIC, Sevilla, Spain)

Thomas M. Lewinsohn (Universidade Estadual de Campinas, São Paulo, Brazil

Jane Memmott (University of Bristol, Bristol, UK)

Jens M. Olesen (University of Aarhus, Aarhus, Denmark)

Theodora Petanidou (University of the Aegean, Mytilene, Greece)

Victor Rico-Gray (Instituto de Ecologia, A.C., Xalapa, Mexico)

John N. Thompson (University of California, Santa Cruz, California, USA) 